\title{
Early childhood language memory in the speech perception of international adoptees*
}

\author{
JANET S. OH \\ California State University, Northridge \\ TERRY KIT-FONG A U \\ The University of Hong Kong \\ AND \\ SUN-AH JUN \\ University of California, Los Angeles
}

(Received 6 March 2009 -Revised I Fuly 2009 -First published online 2 December 2009)

\section{ABSTRACT}

It is as yet unclear whether the benefits of early linguistic experiences can be maintained without at least some minimal continued exposure to the language. This study compared $\mathrm{r} 2$ adults adopted from Korea to the US as young children (all but one prior to age one year) to I 3 participants who had no prior exposure to Korean to examine whether relearning can aid in accessing early childhood language memory. All 25 participants were recruited and tested during the second week of first-semester college Korean language classes. They completed a language background questionnaire and interview, a childhood slang task and a Korean phoneme identification task. Results revealed an advantage for adoptee participants in identifying some Korean phonemes, suggesting that some components of early childhood language memory can remain intact despite many years of disuse, and that relearning a language can help in accessing such a memory.

[*] This work was supported by a grant from the University of Hong Kong (Au). The preparation of this paper was supported by a grant from the National Science Foundation (Oh). We would like to thank Rich Lee, Hangtae Cho, Kim Park Gregg, Marissa Weyer, Sahyang Kim, Timothy Arbisi-Kelm and Kate Bell for their help with this study. These data were presented at the Boston University Conference on Language Development, November 2008. Address for correspondence: Janet Oh, Department of Psychology, California State University, Northridge, i 8 I I I Nordhoff Street, Northridge, CA 9I $330-$ 8255. e-mail: janetoh@alum.pomona.edu 
Without early linguistic experience, it is difficult to acquire native-like phonological abilities (e.g. first language: Fromkin, Krashen, Curtiss, Rigler \& Rigler, I974; Koluchova, I972; I976; second language: Johnson \& Newport, I989; Oyama, I976). There appear to be long-lasting benefits of such early linguistic experience even when regular experience with a language is limited to just the early years, as long as there is at least some minimal continued exposure to the language. For example, English-monolingual adults learning Hindi who had regular exposure to Hindi just during their first two years of life were much better at distinguishing among Hindi speech sounds than their peers who had no such prior experience with the language (Tees \& Werker, I984). Because these adults grew up in Hindi-Canadian communities, they probably continued to hear Hindi phonemes if family or community members spoke Hindi around them and/or if first-generation immigrants in the community spoke in Hindi-accented English.

Similarly, Korean-American adults who had been childhood speakers of Korean (spoke Korean regularly until about age five years) outperformed novice adult learners of Korean in distinguishing among Korean stop consonants (Oh, Jun, Knightly \& Au, 2003). Childhood speakers were also more native-like in their production of those speech sounds. Furthermore, Latino childhood overhearers and childhood speakers of Spanish who were relearning the language as adults spoke Spanish with more native-like accents than their classmates who had no regular exposure to Spanish until learning the language in adolescence (Au, Knightly, Jun \& Oh, 2002; $\mathrm{Au}, \mathrm{Oh}$, Knightly, Jun \& Romo, 2008; Knightly, Jun, Oh \& Au, 2003). As with the Hindi-Canadian language learners, these Korean-American and Latino heritage language learners probably continued to hear the phonemes of their heritage language in the speech of their family and heritage community, and this continued exposure to the language, albeit minimal, may have served to maintain the benefits of their early experiences with the language.

Without at least minimal continued exposure to the target language, linguistic experiences limited to the early years appear to be inaccessible in adulthood. Monolingual French adults who were completely cut off from their childhood language after being adopted from Korea to France between ages three and eight or nine years seemed to show no memory for their childhood language. They were no better than their non-adoptee monolingual French peers on a variety of tasks assessing Korean linguistic ability, including a sentence identification task, a word recognition task, a speech segment detection task and a phoneme discrimination task (Pallier et al., 2003; Ventureyra, Pallier \& Yoo, 2004). Furthermore, adoptees' event-related fMRI activation patterns while listening to Korean and French did not differ from other French monolinguals, nor did the adoptees' patterns differ while listening to an unfamiliar language (Polish) vs. Korean (Pallier et al., 2003). 
In this study, we examine whether the benefits of early linguistic experiences that are limited just to the early years can be maintained without continued exposure to the language. Although it makes intuitive sense that one would have to have some continued experience to maintain early childhood language memory, it is also possible that one could access such early language memory through the process of relearning. In fact, relearning has been found to be an effective way to build up retrieval strength to access a long-ago memory (Bjork \& Bjork, I996; Bjork \& Bjork, 2006). Perhaps early childhood language memory becomes inaccessible without continued exposure, but remains intact. The process of relearning could then help build up retrieval strength to access that memory. Note that while the Korean adoptees were not relearning their childhood language (with the exception of two adoptee participants; see Ventureyra et al., 2004), the adult heritage language learners in the other studies were. The heritage language learners may have been able to access their childhood language memory by relearning the language, whereas the adoptees' childhood language memory may have been harder to access as a result of years of disuse. If the Korean adoptees in France were to relearn Korean as adults, they might show an advantage over novice learners of the language.

To better understand the accessibility of early linguistic memory, this study compared Korean international adoptees relearning Korean as adults with novice adult learners of Korean. If early childhood language memory can be re-activated and accessed upon relearning, then adoptees - who had no experience with Korean after adoption-should nonetheless, upon relearning Korean, show an advantage over novice learners in their perception of Korean phonemes. Moreover, because adoptees in our study were adopted during their first year of life, with one exception, likely before they were speaking, this study also addresses possible lasting benefits of perceptual learning from mere exposure to a language during infancy. This study therefore examined the nature of very early childhood language memory. Specifically, we predicted that childhood language memory will remain intact despite many years of disuse. We also predicted that relearning a childhood language will help in accessing a seemingly lost language memory.

\section{METHOD}

\section{Participants}

Twenty-five participants were recruited from a large university in Minnesota, USA. All participants were enrolled in a first-semester Korean language class that met five days a week for 50 minutes. Twelve participants had been adopted as young children from Korea to Minnesota (mean age = 2I;0; range: 18 to 33 years; 9 female), and $\mathrm{I} 3$ were novice learners of Korean (mean age $=20 ; 2$; range : 18 to 25 years; 6 female). Eleven adoptee participants had 
been adopted by age one year (between 3 months and I year; mean age at adoption: 5 months); one was adopted at age three years.

\section{Materials and procedure}

Data were collected during the second week of the Korean language course. Each participant completed a language background questionnaire, then a Korean language abilities test, and then a follow-up interview. The computerized language abilities test was programmed in $e$-Prime (Schneider, Eschmann \& Zuccolotto, 2002) and included a childhood slang task and a phoneme identification task.

Language background assessment. The language background questionnaire asked about the participant's general language background (e.g. participant's first language, languages spoken by parents) and specific questions about the participant's experiences with Korean since birth. The latter set of questions included both the quantity (how much they heard, were spoken to and spoke Korean) and quality (words/short phrases/sentences; extent of mixing with English) of their linguistic experiences during various periods of their lives. A follow-up interview was conducted to clarify questionnaire responses. In our prior research, we have found that independent reports from informants who knew the participants as young children largely confirmed participants' responses on the language background questionnaire (Au et al., 2002).

To corroborate participants' self-reports of childhood experiences with Korean, we assessed their knowledge of Korean childhood slang. The target slang terms are commonly used around young children at home or on the playground, but are not typically taught in the language classroom (e.g. 'cry-baby', 'spoiled'), and should therefore be a good measure of early childhood experience with Korean. The task is designed to distinguish between those who have had early childhood experience with Korean and those who have not had any such early experience and should be difficult for all participants in this study. Because nearly all adoptee participants were adopted as infants, any knowledge of such childhood slang would likely reflect post-adoption experiences, if any, with Korean. We assessed all participants' slang production and comprehension. To assess slang production, I 4 terms were presented on a computer screen in English, and participants translated them aloud into Korean. For slang comprehension, they heard I 4 slang terms in Korean over headphones and translated them aloud into English. Responses were audiotaped and compared against a list of acceptable responses compiled by Korean-English bilingual research assistants. In previous research, this task reliably corroborated self-reports of language background (Oh et al., 2003).

Phoneme perception. Target phonemes in the phoneme identification task were the three denti-alveolar (lenis: $/ \mathrm{t} /$, tense: $/ \mathrm{t}^{*} /$, aspirated $/ \mathrm{t}^{\mathrm{h}} /$ ) and the 
three velar (lenis: $/ \mathrm{k} /$, tense: $/ \mathrm{k}^{*} /$, aspirated: $/ \mathrm{k}^{\mathrm{h}} /$ ) Korean stop consonants. Korean utilizes a three-way distinction in stop consonants (lenis, tense, aspirated), while English utilizes a two-way distinction (voiced, voiceless). The Korean three-way distinction is therefore usually very challenging for first-time adult learners of Korean. We identified 6 minimal triplets of Korean words that varied only on the target consonant: 3 started with the denti-alveolar consonants (e.g. $/ \mathrm{tal} /, / \mathrm{t}^{*} \mathrm{al} /, / \mathrm{t}^{\mathrm{h}} \mathrm{al} /$ ) and 3 with the velar consonants (e.g. /kong/, / $\mathrm{k}^{*}$ ong/, $/ \mathrm{k}^{\mathrm{h}}$ ong/).

In the phoneme identification task, participants heard one speaker saying two different words (A and B), and then a second speaker saying one of the first two words (X). They were then asked to identify whether X matched A or B (ABX technique; Harris, I 952; Liberman, Harris, Hoffman \& Griffith, I 957). A and B always came from one triplet, and therefore varied only in the target consonant. Each participant completed the phoneme identification task once with the target phoneme in phrase-initial position (target word only) and once in phrase-medial position (target word preceded by /i/, which means 'this'). They indicated their answer by pushing the appropriate button on a button box connected to the computer. Note that this is a very easy task for native Korean speakers; in a previous study, native Korean speakers correctly identified $98.6 \%$ of phonemes in a similar task (target phonemes were the Korean denti-alveolar stop consonants; Oh et al., 2003).

\section{RESULTS}

Language background assessment. All of the adoptee participants were monolingual English speakers. Of the I2 adoptee participants, one was adopted at age three years and could speak Korean then. The only postadoption experience this participant had until the college Korean language class was a summer program focused mostly on Korean culture with some Korean language instruction around age seven or eight years. The remaining I I adoptees were all adopted by age one year (eight were adopted by 6 months). Five of them had no post-adoption experience with Korean until their college Korean language class. Six had minimal exposure (2 during adolescence and 4 during childhood), usually in culture camps for Korean adoptees. These culture camps take place in Minnesota and primarily provide a place for Korean adoptees to socialize and meet other adoptees and their families. They are conducted in English, and some provide minimal Korean language instruction as part of their curriculum; for example, teaching some basic Korean vocabulary words (e.g. counting).

Most of the novice learners were monolingual English speakers. Four novice learners reported that their first language was a non-English language (Chinese, Vietnamese and Cambodian), but none of these languages are in the same language family as Korean, and therefore do not utilize the three-way 
OH ETAL.

TABLE I. Mean accuracy (with standard errors) on phoneme perception task by group

\begin{tabular}{lccc}
\hline & $\begin{array}{c}\text { Adoptee full sample } \\
(n=\mathrm{I} 2)\end{array}$ & $\begin{array}{c}\text { Adoptee subsample } \\
(n=7)\end{array}$ & $\begin{array}{c}\text { Novice learners } \\
(n=\mathrm{I} 3)\end{array}$ \\
\hline Aspirated & $0.85(0.03)$ & $0.83(0.04)$ & $0.74(0.05)$ \\
Lenis & $0.85(0.01)$ & $0.85(0.02)$ & $0.70(0.06)$ \\
Tense & $0.75(0.03)$ & $0.74(0.05)$ & $0.77(0.04)$ \\
\hline
\end{tabular}

a: Adoptee subsample: adoptees who did not have post-adoption experience with Korean during early childhood.

stop consonant distinction of interest (i.e. lenis, tense, aspirated). Nine of the I 3 novice learners reported some minimal prior experience with Korean (5 during adolescence and 4 in adulthood): either watching Korean television or occasionally hearing a few words from a Korean friend.

Performance on the childhood slang task corroborated participants' self-reports of prior experience with Korean. As expected, most participants did not correctly translate any of the slang items. On average, adoptees correctly translated $\mathrm{r} \cdot 8 \%$ of slang production items and $\mathrm{r} \cdot 6 \%$ of slang comprehension items. Novice learners did not correctly translate any item.

Phoneme perception. The phoneme identification task revealed no advantage for adoptees over novice learners in overall accuracy $(t(23)=\mathrm{I} \cdot 67$, n.s., Cohen's $d=0.67$ ) (in all cases, we report one-tailed, planned $t$-tests; see Table I, column i for means). However, adoptees do seem to have an advantage over novice learners on some phonemes, namely, lenis $(t(23)=$ 2.55, $p=0.009, d=\mathrm{I} \cdot 02)$, and aspirated consonants $(t(23)=\mathrm{I} \cdot 83, p=0.04$, $d=0 \cdot 74)$, but not tense consonants $(t(23)=0 \cdot 39$, n.s., $d=0 \cdot 16)$. In order to examine whether there were gender differences in phoneme perception, we also conducted $2 \times 2$ ANOVAs with group (adoptee vs. novice learner) and gender as independent variables. In all cases, for overall phoneme perception and for perception in each phoneme category, there were no reliable main effects of gender $\left(F_{\mathrm{s}}(\mathrm{I}, 2 \mathrm{I})=0 \cdot \mathrm{I}_{\mathrm{I}}\right.$ to $\mathrm{I} \cdot 8 \mathrm{o}$, n.s. $)$, nor were there any reliable group $\times$ gender interactions $(F \mathrm{~s}(\mathrm{I}, 2 \mathrm{I})=0 \cdot 20$ to $\mathrm{I} \cdot 26$, n.s. $)$.

We also re-ran all of the analyses with only those adoptees who were adopted before age one year and had no post-adoption experience with Korean during early childhood. In all cases, the pattern of results was the same (see Table I, column 2). Additionally, in order to examine whether performance on the task was related to age at adoption, we ran bivariate Pearson correlations between age at adoption and accuracy in identifying each of the three types of phonemes. These analyses revealed that age at adoption was not reliably correlated to accuracy in phoneme identification in any category $(r \mathrm{~s}=-0 . \mathrm{I} 8$ to 0.03 , n.s.) (see Figure I). 


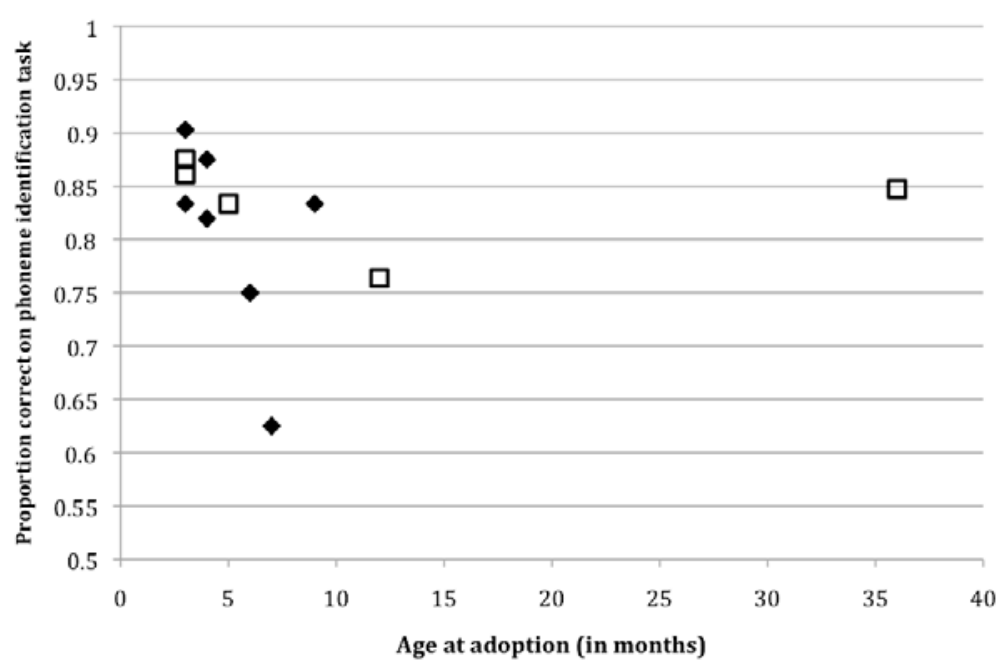

Fig. I. Adoptee participants' overall accuracy on phoneme identification task by age at adoption.

NOTE: Squares represent those adoptee participants who had some post-adoption exposure to Korean in early childhood.

\section{DISCUSSION}

Adoptee participants in this study reliably outperformed novice learners of Korean in identifying some Korean phonemes, even though the adoptees did not have any regular exposure to Korean since being adopted from Korea. Importantly, I I of the I 2 adoptees were adopted during their first year of life. Some components of very early childhood language memory, then, can remain intact even without continued exposure to the language. Although both our adoptee participants and those in Pallier and colleagues' studies were cut off from their childhood language after adoption, the adoptee participants in this study were able to access their early childhood language memory to show an advantage over novice learners in Korean phoneme perception. These findings are noteworthy, given that the Korean adoptees in Pallier et al.'s (2003) study had been adopted at much older ages (between ages three and eight years) than the adoptees in the current study. A potentially important difference between these two samples, namely two weeks of relearning of the Korean language in our sample and no relearning in Pallier and colleagues' sample, calls for further exploration of relearning as a mechanism for re-activating early childhood language memory.

Although there may be other potentially important differences between the samples, the difference in relearning experience has emerged as a particularly 
interesting one. Although speculative at this point, relearning a language may help in accessing a long-ago language memory-especially for the overlearned aspects of language such as phoneme perception. Researchers have proposed a distinction between storage and retrieval strength in memory (Bjork \& Bjork, I 996 ; Bjork \& Bjork, 2006). While storage strength depends on how well something was originally learned, retrieval strength depends on current usage. For a long-ago memory such as adoptees' childhood language memory, storage strength would have been maintained from the original linguistic input, but retrieval strength would be very low due to lack of use. As a result, childhood language memory may seem inaccessible (as in Pallier and colleagues' studies of Korean adoptees). In these cases, relearning can aid in building up retrieval strength for the memory, making the memory accessible as a result. Our findings appear to support this theory.

We recognize that it is also possible that adoptees were able to access their childhood language memory before relearning occurred. That is, adoptee participants may have outperformed novice learners on Korean phoneme perception even before the start of the Korean class, and so the adoptees' advantage may not have been a result of relearning per se. Because we did not collect data before the Korean language class started, we cannot rule out this possibility. However, given the difference between our findings and Pallier and colleagues' findings (Pallier et al., 2003; Ventureyra et al., 2004), we believe that there is a good possibility that adoptees' advantage in this study was due to relearning.

The results of our study also underscore the importance of early linguistic experiences. As much previous research has demonstrated, such early experience is critical for achieving native-like abilities in a language (e.g. Fromkin et al., I974; Koluchova, I972; I976). In fact, infants go from being interested in contrasts between both non-native and native phonemes to only being interested in contrasts between native phonemes during the course of the first year (Werker, I989). Our study demonstrates the long-lasting benefits of such early perceptual learning of language-specific phoneme categories, even when such experience is discontinued for many years thereafter.

Our findings also speak to adults' access to memory from infancy in the domain of perceptual learning. Note that for I I of the $\mathbf{2} 2$ adoptees in our study, early exposure to Korean was limited to their first year of life, most likely before they learned to speak. As infants, they began to learn to categorize Korean phonemes from hearing the ambient language, retained the memory for about two decades and successfully accessed it after less than two weeks of relearning. These findings can have important implications for the long-term retention of perceptual learning. 
We are mindful that adoptee participants did not show an across-the-board advantage. They outperformed novice learners on lenis and aspirated consonants but not on tense consonants. The reason for this is not entirely clear, but it could be due to the two-way vs. three-way laryngeal contrast between English and Korean and how these contrasts correspond to each other. In English, voiceless consonants are aspirated while voiced consonants are not. In Korean, however, both lenis and aspirated consonants are aspirated while tense consonants are not (Korean three-way contrasts also vary in other laryngeal features such as pitch and voice quality at vowel onset; Cho, Jun \& Ladefoged, 2002). That is, lenis and aspirated consonants in Korean belong to the same category as English voiceless consonants in terms of voice onset time. Thus, distinguishing lenis from aspirated consonants in Korean would require attending to other features, making it difficult for native speakers of English.

We focused on phoneme perception because most adoptees in our study had been adopted before learning to speak Korean. In previous studies, we have found that with mere exposure to a language in childhood, childhood overhearers of Spanish spoke Spanish with more native-like phonology than novice learners (Au et al., 2002; Knightly et al., 2003). It remains to be seen whether international adoptees, even if adopted before they were speaking and without continued exposure to their childhood language, could have an advantage in phoneme production as well.

Future studies should also investigate the influence of other factors on the adoptees' advantage. Although we did not find any association between age at adoption and accuracy in phoneme identification, perhaps a sample with a wider adoption age range would uncover a reliable adoption-age effect. More generally, a better understanding of the accessibility of early childhood language memory could not only inform us about a basic mechanism of language acquisition, namely the retention of long-ago childhood memory, it could also help adoptees and adoptive parents to make more informed decisions about heritage language learning.

Previous research had indicated that continued exposure to a language was necessary to maintain the benefits of early linguistic experience. Earlier studies of international adoptees who had made a clean break from their childhood language uncovered no benefits of such early linguistic experience. In contrast, the studies of heritage-language (re)learners who had some minimal exposure to the target language beyond childhood documented reliable and measurable benefits. The present study of international adoptees who were relearning the language as adults suggests that some components of childhood language memory - acquired in early infancy - can remain intact despite many years without exposure to the language, and that relearning the language may help re-activate and access that memory. 


\section{REFERENCES}

Au, T. K., Knightly, L. M., Jun, S.-A. \& Oh, J. S. (2002). Overhearing a language during childhood. Psychological Science 13, 238-43.

$\mathrm{Au}$, T. K., Oh, J. S., Knightly, L. M., Jun, S.-A. \& Romo, L. F. (2008). Salvaging a childhood language. Fournal of Memory and Language 58, 998-гог I.

Bjork, E. L. \& Bjork, R. A. (1996). Continuing influences of to-be-forgotten information. Consciousness and Cognition 5, 176-96.

Bjork, R. A. \& Bjork, E. L. (2006). Optimizing treatment and instruction: Implications of a new theory of disuse. In L.-G. Nilsson \& N. Ohta (eds), Memory and society: Psychological perspectives, ro9-133. New York, NY: Psychology Press.

Cho, T., Jun, S.-A. \& Ladefoged, P. (2002). Acoustic and aerodynamic correlates of Korean stops and fricatives. Fournal of Phonetics 30, 193-228.

Fromkin, V., Krashen, S., Curtiss, S., Rigler, D. \& Rigler, M. (I 974). The development of language in Genie: A case of language acquisition beyond the 'critical period'. Brain and Language $\mathbf{1}, 8 \mathrm{I}-107$.

Harris, J. D. (1952). Remarks on the determination of a differential threshold by the so-called ABX technique. Fournal of the Acoustical Society of America 24, 4I7.

Johnson, J. S. \& Newport, E. L. ( 1989 ). Critical period effects in second language learning: The influence of maturational state on the acquisition of English as a second language. Cognitive Psychology 21, 60-99.

Knightly, L. M., Jun, S.-A., Oh, J. S. \& Au, T. K. (2003). Production benefits of childhood overhearing. Fournal of the Acoustical Society of America I I4, 465-74.

Koluchova, J. (1972). Severe deprivation in twins: A case study. Fournal of Child Psychology and Psychiatry I3, I07-I I4.

Koluchova, J. (1976). The further development of twins after severe and prolonged deprivation: A second report. Fournal of Child Psychology and Psychiatry I7, I8I-88.

Liberman, A. M., Harris, K. S., Hoffman, H. S. \& Griffith, B. C. (I957). The discrimination of speech sounds within and across phoneme boundaries. Fournal of Experimental Psychology 54, 358-68.

Oh, J. S., Jun, S.-A., Knightly, L. M. \& Au, T. K. (2003). Holding on to childhood language memory. Cognition 86, B53-B64.

Oyama, S. ( 1976 ). A sensitive period for the acquisition of a nonnative phonological system. Fournal of Psycholinguistic Research 5, $26 \mathrm{I}-83$.

Pallier, C., Dehaene, S., Poline, J.-B., LeBihan, D., Argenti, A.-M., Dupoux, E. \& Mehler, J. (2003). Brain imaging of language plasticity in adopted adults: Can a second language replace the first? Cerebral Cortex $\mathbf{1 3}$, I 55-61.

Schneider, W., Eschmann, A. \& Zuccolotto, A. (2002). E-Prime user's guide. Pittsburgh, PA: Psychology Software Tools, Inc.

Tees, R. C. \& Werker, J. F. (I984). Perceptual flexibility: Maintenance or recovery of the ability to discriminate non-native speech sounds. Canadian Fournal of Psychology 38, $579-90$.

Ventureyra, V. A. G., Pallier, C. \& Yoo, H.-Y. (2004). The loss of first language phonetic perception in adopted Koreans. Fournal of Neurolinguistics 17, 79-9I.

Werker, J. F. (1989). Becoming a native listener: A developmental perspective on human speech perception. American Scientist 77, 54-59. 